\title{
A decade of pediatric mechanical circulatory support before and after cardiac transplantation
}

\author{
Jonathan M. Chen, MD, ${ }^{\text {a }}$ Marc E. Richmond, MD, MS, ${ }^{\mathrm{b}}$ Kevin Charette, CCP, ${ }^{\mathrm{b}}$ Hiroo Takayama, MD, \\ Mathew Williams, MD, ${ }^{\mathrm{b}}$ Lisa Gilmore, NP, ${ }^{\mathrm{b}}$ Alejandro Garcia, MD, ${ }^{\mathrm{b}}$ and Linda J. Addonizio, $\mathrm{MD}^{\mathrm{b}}$
}

Objectives: We reviewed the use of pediatric mechanical circulatory support before and after transplantation to examinine current results and future strategies.

\begin{abstract}
Methods: All patients listed for transplantation from January 2000 to December 2010 who required either extracorporeal membrane oxygenation (ECMO) or ventricular assist device (VAD) support before ("intention to transplant") or after transplantation were included. Indications for mechanical assistance, age, weight, duration of support, complications while on support, causes of death, and overall actuarial survival were recorded.
\end{abstract}

\begin{abstract}
Results: Thirty-seven patients were received VADs; 32 (86.5\%) survived to transplantation. Postoperative hemorrhagic or thrombotic complications affected all of those under $15 \mathrm{~kg}$. One patient in the survivor cohort demonstrated focal neurologic findings. Three $(8.1 \%$ ) had panel reactive antibody levels of $10 \%$ or more while on device support; all received transplants. ECMO as an intention to bridge to transplantation was used in 28 patients; 7 died, 7 were weaned, and 14 were bridged to transplantation. Nineteen patients required ECMO after transplantation; 3 additional patients had percutaneous VAD support for late rejection. There was a significant $(P=.02)$ difference in survival after listing for transplantation among those supported with ECMO, with VAD, and those not supported with a device. No difference in posttransplant survival was demonstrated between those patients supported with either ECMO or VAD before transplant compared with all others not bridged to transplantation.
\end{abstract}

Conclusions: Both VAD and ECMO support are highly effective means of bridging patients to transplantation and supporting patients after transplanatation. Ideally, the availability of smaller devices for children will have a favorable impact on the morbidity related to anticoagulation in the smallest patients. (J Thorac Cardiovasc Surg 2012;143:344-51)

Over the past 2 decades, the use of ventricular assist devices (VADs) as bridges to transplantation in adults has become the standard of care worldwide. However, VAD use in children in particular the specific choice of device and method of support continues to be controversial. Until recently, extracorporeal membrane oxygenation (ECMO) represented the mainstay of mechanical ventricular assistance in pediatric patients, particularly for those considered "too small for VAD." The emergence of new, smaller devices more appropriate for children has made available a wider variety of candidate devices, albeit still with considerable hemorrhagic and thromboembolic morbidity for those less than $10 \mathrm{~kg}^{1-5}$ For pediatric patients being bridged to transplantation, the device choice is generally

From Weill Medical College of Cornell University ${ }^{\mathrm{a}}$ and College of Physicians and Surgeons of Columbia University, ${ }^{\mathrm{b}}$ New York, NY.

Disclosures: Dr Williams reports the following: Edwards, proctoring TAVI (Consultant); Medtronic, consulting (Consultant); St. Jude, consulting (Consultant).

Read at the 91st Annual Meeting of The American Association for Thoracic Surgery, Philadelphia, Pennsylvania, May 7-11, 2011.

Received for publication May 7, 2011; revisions received Sept 9, 2011; accepted for publication Oct 25, 2011; available ahead of print Dec 6, 2011.

Address for reprints: Jonathan M. Chen, MD, 525 East 68th St-Suite M404, New York, NY 10021 (E-mail: Jmc23@columbia.edu).

$0022-5223 / \$ 36.00$

Copyright (c) 2012 by The American Association for Thoracic Surgery doi:10.1016/j.jtcvs.2011.10.072 influenced by patient size, the estimated duration of support, and the relative feasibility for home discharge. In contrast, after transplant, the increased risk of significant infection and the potential morbidity associated with reoperative sternotomy may influence device choice considerably. We sought to review our single-center experience over a decade with the use of a variety of VAD types and ECMO in children both as an intended bridge to transplant and for temporary support in the setting of salvage therapy for transplant rejection, with the aim of examining current results and future strategies.

\section{METHODS}

All patients listed for transplantation at the Morgan Stanley Children's Hospital of New York Presbyterian from January 1, 2000, to December 31, 2010, were reviewed, and those undergoing either ECMO or any form of VAD support before ("intention to transplant") or after transplantation were included for analysis. Some of those who received posttransplant sup port during this 10 year window initially underwent transplantation before 2000. In addition, 6 children who underwent placement of VADs at other institutions but who were later managed and underwent transplantation at our hospital were also included in the analysis.

Indications for mechanical assistance, age, weight, duration of device support, complications while on device support (device exchange, reopera tion, embolic or hemorrhagic complications, neurologic complications), panel reactive antibody levels, and indicators of end organ function (creat inine, aspartate transaminase [AST], alanine transaminase [ALT]), the need 


\section{Abbreviations and Acronyms \\ ALT = alanine transaminase \\ AST $=$ aspartate transaminase \\ $\mathrm{BTT}=$ bridge to transplantation (cohort) \\ ECMO $=$ extracorporeal membrane oxygenation \\ LVAD $=$ left ventricular assist device \\ POD = postoperative day \\ PTT = partial thromboplastin time \\ PVAD $=$ paracorporeal ventricular assist device \\ RVAD $=$ right ventricular assist device \\ $\mathrm{VAD}=$ ventricular assist device}

for postoperative renal replacement therapy and overall outcome (weaned, transitioned to a different form of support, transplant, retransplant, death), causes of death, and overall actuarial survival were recorded. Post VAD end organ function data were obtained 1 week postoperatively where pos sible, and for those supported for less than 1 week, at 72 hours after implant; 1 patient on support for only 24 hours and all 6 patients who underwent VAD implantation at another hospital center were excluded from end organ func tion data analysis. Panel "reactivity" was classified as positive if either class I or II panel reactive antibody was $10 \%$ or more by complement mediated cytotoxicity assay in the presence of dithiothreitol.

Patients experiencing hemodynamic compromise within the first 30 days after transplantation were considered to exhibit primary graft failure (rather than acute rejection). "Late" rejection was defined as occurring more than 1 year postoperatively.

\section{Management of Anticoagulation}

Perioperative anticoagulation was managed by a multidisciplinary team and for those patients more than $15 \mathrm{~kg}$ involved the commencement of un fractionated heparin within the first 24 to 48 hours of the operating room, once the chest tube output had decreased below 1 to $2 \mathrm{~mL} \cdot \mathrm{kg}^{1} \cdot \mathrm{h}^{1}$, ulti mately targeting a partial thromboplastin time (PTT) of 60 to 80 seconds. Of this cohort, those patients managed with the Thoratec paracorporeal VAD (PVAD; Thoratec Corporation, Pleasanton, Calif), HeartMate II (Thermo Cardiosystems, Inc, Woburn, Mass), and Toyobo (Nipro Corpora tion, Osaka, Japan) were subsequently transitioned to oral warfarin therapy with a goal international normalized ratio of 2.5 to 3.0 seconds. Patients with the Levitronix CentriMag (Thoratec Corporation, Pleasanton, Calif), Abiomed BVS 5000 (Abiomed, Danvers, Mass), and Berlin Heart EXCOR (Berlin Heart GmbH, Berlin, Germany) remained on heparin alone. For those patients weighing less than $15 \mathrm{~kg}$, our protocol evolved to ultimately beginning unfractionated heparin in the operating room before transport, with a goal PTT of 80 to 100 seconds. Additional aspirin and dipyridamole were begun once patients were tolerating oral therapy. Patients on the HeartMate XVE VAD received no heparin therapy and only aspirin.

Those exhibiting prothrombotic tendencies and those with difficulty achieving goal anticoagulation were managed under the direction of a pedi atric hematologist. Neither activated clotting time nor thromboelastogra phy was used postoperative to guide anticoagulation protocols.

\section{Percutaneous Support}

In the cardiac catheterization laboratory, an $8 \mathrm{~mm}$ Dacron graft was sewn end to side to the axillary artery and an Abiomed Impella LP was in serted under fluoroscopic guidance. A Swan Ganz catheter (Edwards Life Sciences, Irvine, Calif) was placed via the internal jugular veins and subsequently replaced with a stiff guidewire (Meier guidewire, Boston Sci entific, Natick, Mass), and a BioMedicus (Medtronic, Inc, Minneapolis,
Minn) femoral venous cannula was introduced leaving its tip in the main pulmonary artery. A second femoral venous cannula was placed via the common femoral vein and adjusted with its tip in the right atrium for right VAD (RVAD) inflow. These latter 2 cannulas were connected to a Centri Mag circuit (Thoratec) and both left and right sided devices actuated.

\section{Technical Modifications}

Early in the experience, all VAD cases were performed on cardiopulmo nary bypass, and every attempt was made to close intracardiac communica tions to prevent postoperative right to left shunting. This strategy evolved to performing some VAD implants off bypass when possible, and even in those pediatric patients with significant intracardiac communications (including multiple ventricular septal defects) there was no significant difficulty "bal ancing" shunting, nor were there increased neurologic sequelae.

Additionally, in the early experience, the patients supported by the Cen triMag device who experienced thrombotic complications were supported by the "adult" CentriMag circuit ( $3 / 8$ inch tubing and hard connectors) although some of these complications were ameliorated by using $3 / 8$ to $1 / 4$ inch X coated taper tubing (Terumo Cardiovascular, Ann Arbor, Mich). For children $10 \mathrm{~kg}$ and under, we currently use the PediMag device (Thoratec), which requires higher revolutions per minute to achieve com parable flow, $1 / 4$ inch tubing, and connectors.

The ECMO circuit used from January 2000 through October 2007 con sisted of a Jostra HL 20 (Maquet, Inc, Wayne, NJ) roller pump with $1 / 2$ inch or 3/8 inch raceway tubing, a Medtronic silicone 2500 or 3500 oxygenator (Medtronic, Inc), an arteriovenous bridge, and several pigtails and dia monds. The total prime of this circuit was approximately $1000 \mathrm{~mL}$. Since October 2007, the ECMO circuit has consisted of 3/8 inch tubing, a Rota flow (Maquet, Inc) centrifugal head, a Quadrox D (Maquet, Inc) oxygenator, and no arteriovenous bridge, pigtails, or diamonds. The total prime of the contemporary circuit is $360 \mathrm{~mL}$. In addition, 1 patient underwent ECMO af ter transplantation for primary graft failure using a PediMag pump, a Quad rox Pediatric iD oxygenator, and $1 / 4$ inch tubing and connectors throughout.

\section{Statistical Analysis}

All continuous variables are expressed as mean \pm standard deviation. Preoperative and postoperative laboratory values were compared by using a paired Student $t$ test. Comparisons between groups were made using a 2 sample Student $t$ test. For survival from listing and transplant, Kaplan Meier survival analysis was used and cumulative survival graphs created. Comparisons of survival between groups were made using the $\log$ rank test. All statistical tests were 2 sided. Statistical analyses were performed using SPSS Statistics 18 for Windows (IBM Corp, Somers, NY). Patients undergoing ECMO bridge to VAD support were included in the "VAD" cohort for actuarial survival analysis.

This study was approved by the Institutional Review Board of Columbia University.

\section{RESULTS \\ VAD Patient Cohort}

Between January 1, 2000, and December 31, 2010, 37 patients were implanted with VADs as intended bridge to transplantation (BTT cohort). During that time interval, 269 overall pediatric patients were listed for transplantation and 206 patients received a transplant. Over the decade of study, a variety of devices were used (Table 1). Causes of heart failure in the BTT cohort included dilated cardiomyopathy in $28(75.7 \%)$, myocarditis in $4(10.8 \%)$, postchemotherapy cardiomyopathy in $2(5.4 \%)$, restrictive cardiomoypathy in $1(2.7 \%)$, peripartum cardiomyopathy in $1(2.7 \%)$, and postcardiotomy shock in $1(2.7 \%)$. One 
TABLE 1. Demographics of the VAD study cohort

\begin{tabular}{lccccc}
\hline \multicolumn{1}{c}{ Device } & n & Age $(\mathbf{y})$ & Weight & Duration (d) & Outcome \\
\hline BVS 5000i & 2 & $12.9 \pm 2.6$ & $53 \pm 1.4$ & $3.0 \pm 2.8$ & 1 Transplant/1 weaned \\
CentriMag BiVAD & 10 & $7.6 \pm 5.0$ & $30.2 \pm 22.3$ & $12.1 \pm 8.4$ & 6 Transplant/4 died \\
PediMag BiVAD & 1 & 0.75 & 5.8 & 17 & 1 Transplant \\
EXCOR BiVAD & 1 & 0.3 & 3.8 & 6 & Died \\
HeartMate (7XVE/2HMII) & 9 & $15.0 \pm 4.0$ & $66.5 \pm 51.8$ & $75.7 \pm 19.9$ & 9 Transplant \\
PVAD BiVAD & 8 & $14 \pm 2.2$ & $58.5 \pm 15.7$ & $43.6 \pm 40.3$ & 8 Transplant \\
Toyobo (1 BiVAD/5 LVAD) & 6 & $13.3 \pm 4.8$ & $33.7 \pm 11.0$ & $313.8 \pm 283.6$ & 6 Transplant \\
CentriMag/Impella & 3 & $14.3 \pm 3.1$ & $56.8 \pm 10.1$ & $5.0 \pm 2.0$ & 3 Weaned \\
\hline
\end{tabular}

BVS 5000i, Abiomed, Danvers, Mass; CentriMag and PediMag, Thoratec Corporation, Pleasanton, Calif; EXCOR, Berlin Heart GmbH, Berlin, Germany; HeartMate, Thermo Cardiosystems, Inc, Woburn, Mass; Toyobo, Nipro Corporation, Osaka, Japan. VAD, ventricular assist device; BiVAD, biventricular assist device; PVAD, paracorporeal ventricular assist device; $L V A D$, left ventricular assist device.

patient initially underwent ECMO support and was later transitioned to an Abiomed (Abiomed, Inc) BVS500i after a Ross procedure. He was successfully weaned from BiVAD support only to require transplant 11 months later. Another 2 patients were transitioned from ECMO to Thoratec PVAD (Thoratec Corporation). Six patients had undergone insertion of a Toyobo (Nipro Corporation) VAD at another institution but received their transplant at our institution. Of these 37 patients in the BTT VAD cohort, 32 (86.5\%) survived to ultimate transplantation.

In the BTT cohort, 5 patients $(13.5 \%)$ died on device support. One patient with a Berlin Heart EXCOR BiVAD required pump exchange and later died of thromboembolic stroke on postoperative day (POD) 6. The remaining 4 underwent Thoratec CentriMag biventricular support; 1 died of progressive thrombotic complications (POD 13), 1 of thromboembolic stroke (POD 27), and 2 of sepsis and multisystem organ failure (POD 14, POD 24). Of note, 3 of these CentriMag patients weighed less than $15 \mathrm{~kg}$.

Postoperative hemorrhage requiring mediastinal exploration affected $7(70 \%)$ of those in the CentriMag cohort, $4(50 \%)$ of those in the Thoratec PVAD cohort, and the 1 patient with Levitronix (Levitronix, Waltham, Mass) PediMag BiVAD support. None of those with the Abiomed BVS and 1 patient (of 9) with a Thoratec HeartMate (XVE or HeartMate II) device had bleeding complications mandating reoperation.

Postoperative thrombotic events requiring device exchange occurred in 3 patients. The EXCOR patient $(3.8 \mathrm{~kg}$ ) required device exchange on POD 4 for thrombus below the RVAD inflow valve; thrombus later developed in both the left and right pumps. The CentriMag patient $(10.8 \mathrm{~kg}) \mathrm{re}-$ quired exchange on POD 1 for clot within the left ventricular assist device (LVAD) inflow tubing; he later received a transplant. One Toyobo patient $(17.0 \mathrm{~kg})$ had a subdural hemorrhage develop in the setting of an elevated international normalized ratio requiring cessation of anticoagulation and neurosurgical intervention. During this period without anticoagulation, a thrombus developed in the device, mandating exchange for a CentriMag system (with later systemic heparinization), which successfully bridged him to transplant.
When analyzed on the basis of patient size, all of those patients less than $15 \mathrm{~kg}$ had either a hemorrhagic or thrombotic complication after VAD implantation.

End-organ dysfunction, as measured by AST/ALT and serum creatinine before and after VAD implantation, demonstrated a trend toward improvement across all parameters: AST $(192.6 \pm 319.7 \mathrm{U} / \mathrm{L}$ before, $90.23 \pm 79.6 \mathrm{U} / \mathrm{L}$ after $)$, ALT (156.5 $\pm 323.6 \mathrm{U} / \mathrm{L}$ before, $72.3 \pm 64.1 \mathrm{U} / \mathrm{L}$ after), creatinine $(1.1 \pm 0.7 \mathrm{mg} / \mathrm{dL}$ before, $0.9 \pm 0.8 \mathrm{mg} / \mathrm{dL}$ after $)$. Perioperative continuous venovenous hemofiltration was required in 7 patients ( 2 of whom died of mutiorgan failure), no patients required hemodialysis, and no patients were permanently dialysis dependent after transplant. One patient (PVAD) in the survivor cohort experienced an acute focal neurologic event while on device support. Although unclear whether his subdural hemorrhage and basal ganglia infarcts represented old watershed events (with conversion in the setting of anticoagulation) or new device-related complications, their clinical manifestation (visual field deficits) have since largely resolved. One patient (Toyobo) had a subdural hemorrhage with headache (described above), and 1 patient (CentriMag) had a critical care neuropathy that resolved. Only $3(8.1 \%)$ patients in the VAD cohort had panel reactive antibody levels of $10 \%$ or greater while on device support. Of these, 1 received intravenous immunoglobulin therapy with plasmapheresis, 1 received intravenous immunoglobulin therapy with mycophenolic mycophenolate mofetil, and 1 received no therapy. All 3 underwent successful transplantation with pre cardiopulmonary bypass exchange transfusions and at last follow-up did not demonstrate panel reactivity.

In addition to the BTT cohort, 3 patients underwent percutaneous VAD support for hemodynamically significant late rejection (age, $14.3 \pm 3.1$ years; weight, $56.8 \pm 10.1 \mathrm{~kg}$; duration of support, $5 \pm 2$ days), and all 3 survived to explant.

\section{ECMO Patient Cohort}

During the study period, 28 patients were placed on ECMO who were listed for transplantation. Of these, $7(25 \%)$ patients died while on support (2 dilated cardiomyopathy, 1 meningitis/shock, 1 myocarditis, and 3 postcardiotomy). 


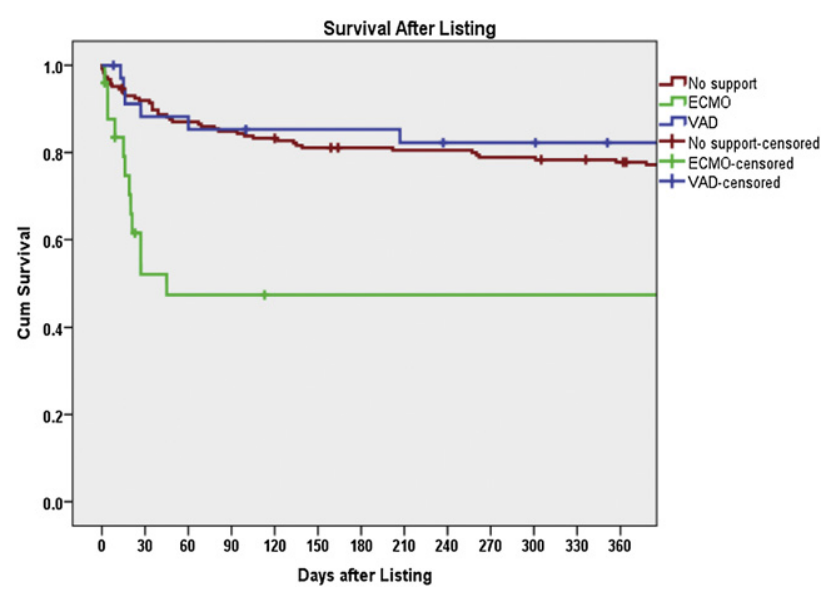

FIGURE 1. Survival after listing. ECMO, Extracorporeal membrane oxygenation; $V A D$, ventricular assist device.

Another $7(25 \%)$ patients were weaned from support without the need for later transplantation, all but 1 of whom had myocarditis (1 newborn infant had cardiogenic shock in the setting of a cryptogenic myocardial infarction). The remaining 14 $(50 \%)$ patients were bridged to transplantation. When analyzed with regard to era of ECMO (pre/post October 2007), survival of this ECMO "BTT" cohort before October 2007 was $68.4 \%$, and after October 2007 was $88.9 \%$.

A total of 19 patients underwent ECMO after transplantation. Of these patients, 10 underwent ECMO support for acute rejection, of whom $3(30 \%)$ died, $6(60 \%)$ were weaned, and $1(10 \%)$ required retransplantation. ECMO support for primary graft failure in the first 2 weeks after transplant was required in 8 patients, of whom $4(50 \%)$ died, $3(37.5 \%)$ were weaned, and $1(12.5 \%)$ required retransplantation. Both cohorts were comparable in duration of support (7.3 \pm 5.0 days vs $6.8 \pm 2.1$ days), although the acute rejection cohort was older $(10.1 \pm 7.6$ years vs $2.6 \pm 3.2$ years $)$ and larger $(30.7 \pm 17.0 \mathrm{~kg}$ vs $9.6 \pm 5.1 \mathrm{~kg})$.

\section{Kaplan-Meier Actuarial Survival}

Kaplan-Meier survival analysis demonstrated a significant $(P=.02)$ difference in actuarial survival after listing for transplantation among those supported with ECMO with VAD and those not supported with a mechanical device (Figure 1) (ECMO vs no support, $P=.001$; ECMO vs VAD, $P=.006$; VAD vs no support, $P=-.515$ ). In contrast, no difference in posttransplant survival was demonstrated between those patients supported with either ECMO or VAD before transplant compared with all others not bridged to transplantation $(P=.84$ (Figure 2).

\section{DISCUSSION}

On the basis of ongoing success in the adult population with VAD therapy as temporary or permanent ventricular

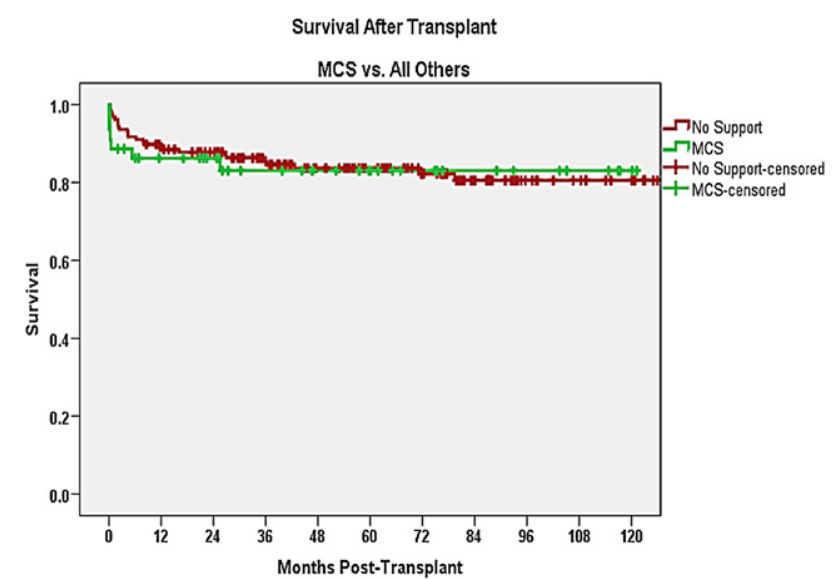

FIGURE 2. Survival after transplant. MCS, Mechanical circulatory support.

support, growing enthusiasm has evolved over the past 20 years to extend the benefit of mechanical ventricular assistance to pediatric patients before and after transplantation. ${ }^{1-6}$ Although initial forays involved simple application of adult-sized devices to older adolescents and teenagers, subsequent adaptation of these devices and the development of new, smaller devices of a size appropriate for small children have offered promising alternatives for increasing numbers of pediatric patients. ${ }^{7}$ However, considerable morbidity still exists with the application of any such devices for infants and small children, in particular related to the difficulty of accurately anticoagulating these patients, and thus the attendant risks of thrombosis, embolism, or significant hemorrhage.

For all transplant recipients, a risk of primary graft failure or rejection with cardiovascular collapse always exists. In selected instances, the need for support may be purely hemodynamic (and not pulmonary), and strategies to avoid the use of an oxygenator in line (ie, VAD not ECMO) may thus be favored. At our center, the recent availability of percutaneous VADs for temporary support has provided logical candidate devices for use in this posttransplant setting.

We sought to review our 10-year experience with the use of different kinds of mechanical ventricular assistance before and after transplantation. In limiting this cohort to those listed for transplantation, we intended to refine a strategy specific to this group and not include those in whom ventricular recovery is likely; for example "simple" postcardiotomy failure where the considerations of device choice based on longer durations of support are less relevant. Our findings are notable for 5 salient findings.

First, VAD therapy can be a highly successful means of supporting pediatric patients across a wide range of sizes. We have tended, in general, to favor biventricular support for patients in whom there is any contention regarding the 
suitability of right ventricular function. However, for those larger patients in whom a long waiting time is projected, where possible we endorse a long-term device with the possibility of home discharge (eg, HeartMate XVE/HeartMate II, PVAD).

Second, bleeding and/or thrombosis remain a major source of morbidity and mortality for patients less than 15 $\mathrm{kg}$ in weight. This particular problem has been highlighted previously by investigators at Great Ormond Street, who implicated, among other reasons, the immaturity of the coagulation cascade in small children. ${ }^{2,7}$ Our management of these smaller patients has evolved considerably over the past decade and now involves several key features: (1) Where possible (unless the possibility of ventricular recovery is high), we use atrial inflow performed with lowdose heparinization off bypass. This has markedly reduced our initial rate of hemorrhage and re-exploration. (2) We use heparin-coated (Carmeda; Medtronic, Inc, Minneapoils, Minn) cannulas and connectors and biocompatible (Smart; Cobe Cardiovascular, Arvada, Colo) tubing. (3) We begin unfractionated heparinization in the operating room before transfer, which is dosed for a goal PTT of 100 to 120 seconds within the first several hours postoperatively. Once extubated, patients are also begun on aspirin and/or warfarin and a second antiplatelet agent once tolerating oral medication. We have not used activated clotting time or thromboelastography to guide our postoperative care owing to specific institutional restrictions.

Using our current strategies of the PediMag device with a smaller (1/4-inch) circuit, we have seen significantly less thrombus (and bleeding events) with the PediMag device in both the bridge-to-transplant setting and for several other nontransplant patients in whom we have used it for postcardiotomy shock (data not shown). ${ }^{8,9}$ While still preliminary, our PediMag experience has been promising with regard to ease of implantation, overall cost, and morbidity; issues of durability and applicability will require further analysis. In the past we have been less inclined to use bridge-totransplant VAD support for multiple reoperative congenital candidates (favoring ECMO). However, using this new approach, and on the basis of recent experience in the literature, we may be more liberal in using this therapy. ${ }^{10}$

Of interest, none of our patients in whom the HeartMate II device was implanted had the development of acquired von Willebrand deficiency, as has been demonstrated extensively in the adult experience. ${ }^{11}$ Although this was fortunate, the possibility of its occurrence remains a consideration for those patients anticipating a long waiting time. As experience grows with the use of this device in children, the magnitude of the acquired von Willebrand phenomenon will become more well defined. ${ }^{12}$ Also of note is our low incidence of acute neurologic morbidities in the entire VAD survivor cohort. However, 3 patients in the nonsurvivor group died of profound neurologic events.
Third, for those bigger patients with refractory late rejection, we have found the percutaneous therapy of Impella LVAD with CentriMag RVAD to be efficacious and cost effective. This approach obviates the morbidity of a reoperative sternotomy, with its attendant risks of both bleeding and infection, the latter being a considerable concern in these patients for whom treatment requires substantial pharmacologic impairment of immune function. This combination VAD is quick to deploy and easy to wean (the sidearm graft can be stapled and the incision closed in the interventional suite). For those smaller patients in this candidate cohort, such a strategy may also avoid significant limb ischemia. We used the Impella 2.5 in this setting given its rapid availability. However, for larger patients this clearly only represents partial support; therefore, the current availability of Impella 5.0 would argue for its use where feasible. Although we supported patients for as long as 1 week with this strategy, should their function have remained poor, consideration of transition to a longer-term VAD would have been necessary. In as much as this approach affords the flexibility to insert an oxygenator into the RVAD limb when necessary, we have favored VAD support over ECMO where possible, a strategy that is substantiated by recent reports of the $50 \%$ success rate of ECMO in this setting. ${ }^{13}$

Our results with posttransplant ECMO support for primary graft failure or rejection mirror those in the literature, likely reflecting the dire clinical scenario warranting its practice. Unlike the Denver group, we have had several survivors supported for more than 4 days ( 1 as long as 19 days) while aggressive antirejection therapy was administered. ${ }^{14,15} \mathrm{We}$ are judicious in the consideration of retransplantation for primary graft failure, especially in the setting of acute rejection, recognizing that outcomes in this setting may be limited.

Fourth, only $8 \%$ of our VAD cohort had significant antiHLA panel reactive antibody levels while on device support, despite the fact that many received multiple blood transfusions. This is in contrast with other recent reports suggesting rates of sensitization as high as $35 \% .{ }^{15}$ We assiduously re-evaluate these levels while on VAD support and currently treat those sensitized patients with the combination of intraoperative exchange transfusion, postoperative plasmapheresis when there is a positive cross match, and maintenance steroids for at least 6 months after transplantation.

Fifth, as has been demonstrated in several multiinstitutional database analyses, our cohort showed no significant survival disadvantage after transplant for those patients who survive to transplantation bridged with either ECMO or VAD (Figure 2). ${ }^{16,17}$ The differences in actuarial survival from the time of listing for transplantation among the cohorts (Figure 1) likely stems from several early deaths in our ECMO group before circuit design change in 2007. Although anecdotal, we believe that the smaller circuit 


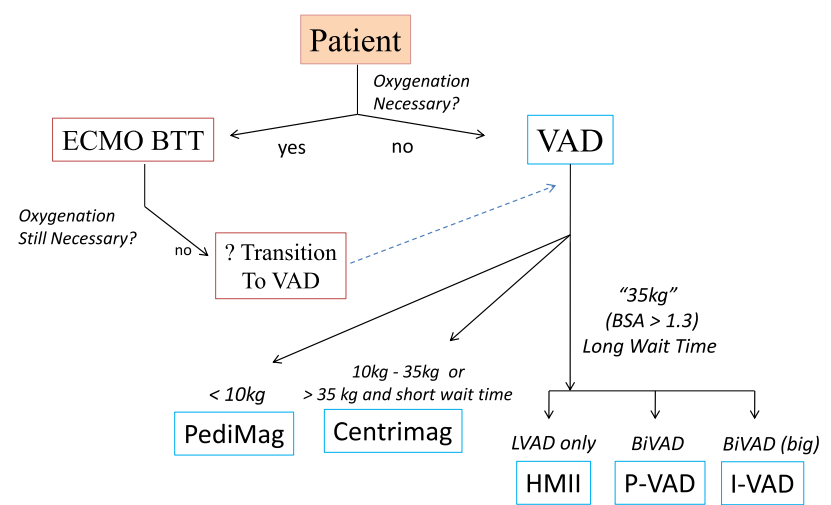

FIGURE 3. Our general approach to mechanical ventricular assistance. $E C M O$, Extracorporeal oxygenation; $B T T$, bridge to transplantation; $V A D$, ventricular assist device; $P V A D$, paracorporeal ventricular assist de vice; $I V A D$, implantable ventricular assist device; $B S A$, body surface area.

prime and elimination of bridges, connectors, and diamonds has reduced some of the morbidity experienced with the earlier circuit.

In summary, we have found it helpful to have a variety of mechanical ventricular assistance available so as to tailor the device strategy to the patient. Although we have no firm algorithm in this setting, our general approach is outlined in Figure 3. Our initial indication for ECMO use pertains to the need for oxygenation, and we favor VAD support where feasible, given (1) the substantial advantages of potential extubation and ambulation/rehabilitation and (2) the disadvantages of having an oxygenator in line (eg, activation of inflammation, increased requirement for anticoagulation, and limited durability). This inclination is in keeping with other reports demonstrating superior survival in patients supported with VAD rather than ECMO.$^{18,19}$ The selection of VAD devices is directed mostly by size and the need for univentricular or biventricular support; however, the optimal strategy to support patients in the "gray zone" of size $(15-35 \mathrm{~kg})$ remains somewhat patientspecific. For those patients in whom neurologic status is in question, we have used a "bridge to decision" approach, initially using a temporary device or ECMO until a more definitive neurologic prognosis can be determined, at which point transition to a longer-term device can take place. Although we have favored using ECMO to support patients with complex congenital heart disease in the past, we would imagine them to be VAD candidates (following this algorithm by size) in many circumstances.

Mechanical circulatory support using a variety of devices is a highly effective means of both bridging patients to transplantation and supporting patients after transplantation during hemodynamic crises. The more widespread availability of smaller and potentially implantable devices for children will only serve to improve these strategies, while potentially affecting favorably the substantial morbidity related to anticoagulation in the smallest patients.

\section{References}

1. Fan Y, Weng YG, Xiao YB, Huebler M, Franz N, Potapov E, et al. Outcomes of ventricular assist device support in young patients with small body surface area. Eur J Cardiothorac Surg. 2011;39:699-704.

2. Brancaccio G, Amodeo A, Ricci Z, Morelli S, Gagliardi MG, Iacobelli R, et al. Mechanical assist device as a bridge to heart transplantation in children less than 10 kilograms. Ann Thorac Surg. 2010;90:58-62.

3. Karimova A, Van Doorn C, Brown K, Giardini A, Kostolny M, Mathias M, et al. Mechanical briding to orthotopic heart transplantation in children weighing less than 10 kg: feasibility and limitations. Eur J Cardiothorac Surg. 2011; 39:304-9.

4. Gandhi SK, Huddleston CB, Balzer DT, Epstein DJ, Boschert TA, Canter CE Biventricular assist devices as a bridge to heart transplantation in small children. Circulation. 2008;118(14 Suppl):S89-93.

5. Morales DL, Almond CS, Jaquiss RD, Rosenthal DN, Naftel DC, Massicotte M, et al. Bridging children of all sizes to cardiac transplantation: the initial multicenter North American experience with the Berlin Heart EXCOR ventricular assist device. J Heart Lung Transplant. 2011;30:1-8.

6. Morales DL, Zafar F, Rossano JW, Salazar JD, Jefferies JL, Graves DE, et al. Use of ventricular assist devices in children across the United States: analysis of 7.5 million pediatric hospitalizations. Ann Thorac Surg. 2010;90: 1313-8.

7. Andrew M, Vegh P, Jonston M, Bowker J, Ofosu F, Mitchell L, et al. Maturation of the hemostatic system during childhoold. Blood. 1992;80:1998-2005.

8. Hirata Y, Charette K, Mosca RS, Quaegebeur JM, Chen JM. Pediatric application of the Thoratec CentriMag BIVAD as a bridge to heart transplantation. $J$ Thorac Cardiovasc Surg. 2008;136:1386-7.

9. Dasse KA, Gellman B, Kameneva MV, Woolley JR, Johnson CA, Gempp T, et al. Assessment of hydraulic performance and biocompatibility of a MagLev centrifugal pump system designed for pediatric cardiac or cardiopulmonary support. ASAIO J. 2007;53:771-7.

10. VanderPluym CJ, Rebeyka IM, Ross DB, Buchholz H. The use of ventricular assist devices in pediatric patients with univentricular hearts. $J$ Thorac Cardiovasc Surg. 2011;141:588-90.

11. Uriel N, Pak SW, Jorde UP, Jude B, Susen S, Vincentelli A, et al. Acquired von Willebrand syndrome after continuous-flow mechanical device support contributes to a high prevalence of bleeding during long-term support and at the time of transplantation. J Am Coll Cardiol. 2010;56:1207-13.

12. Owens WR, Bryant R 3rd, Dreyer WJ, Price JF, Morales DL. Initial clinical experience with the HeartMate II ventricular assist system in a pediatric institution. Artif Organs. 2010;34:600-3.

13. Tissot C, Buckvold S, Phelps CM, Ivy DD, Campbell DN, Mitchell MB, et al Outcome of extracorporeal membrane oxygenation for early primary graft failure after pediatric heart transplantation. J Am Coll Cardiol. 2009;54:730-7.

14. Bae J, Frischer JS, Waich M, Addonizio LJ, Lazar EL, Stolar CJH. Extracorporeal membrane oxygenation in pediatric cardiac transplantation. J Pediatr Surg. 2005;40:1051-7.

15. O'Connor MJ, Menteer J, Chrisant MR, Monos D, Lind C, Levine S, et al. Ventricular assist device-associated anti-human leukocyte antigen antibody sensitization in pediatric patients bridged to heart transplantation. J Heart Lung Transplant. 2010;29:109-16.

16. Jeewa A, Manlhiot C, McCrindle BW, Van Arsdell G, Humpl T, Dipchand AI. Outcomes with ventricular assist device versus extracorporeal membrane oxygenation as a bridge to pediatric heart transplantation. Artif Organs. 2010;34:1087-91.

17. Imamura M, Dossey AM, Prodhan P, Schmitz M, Frazier E, Dyamenahalli U. Bridge to cardiac transplant in children: Berlin Heart versus extracorporeal membrane oxygenation. Ann Thorac Surg. 2009;87:1894-901.

18. Blume ED, Naftel DC, Bastardi HJ, Duncan BW, Kirklin JK, Webber SA. Pediatric Heart Transplant Study Investigators. Outcomes of children bridged to heart transplantation with ventricular assist devices: a multi-institutional study. $\mathrm{Circu}$ lation. 2006;113:2313-9.

19. Davies RR, Russo MJ, Hong KN, O'Byrne ML, Cork DP, Moskowitz AJ, et al. The use of mechanical circulatory support as a bridge to transplantation in pediatric patients: an analysis of the United Network for Organ Sharing database. J Thorac Cardiovasc Surg. 2008;135:421-7.

\section{Discussion}

Dr J. William Gaynor (Philadelphia, $P a$ ). I congratulate Dr Chen and his co investigators on a very interesting presentation and thank them for allowing me to review the manuscript. 
This is an important subject. As we have all seen, increasing numbers of children are listed for cardiac transplantation. In light of the nonincreasing donor pool, waiting times continue to in crease and the potential loss of patients on the waiting list is a sig nificant problem. The findings of this study are very similar to our findings in a similar sized patient cohort, that overall survival to transplant is excellent and that survival after transplant from a VAD is equivalent to that of patients undergoing transplantation without a VAD. I think this is a very effective technique.

We also see the same problems with thrombosis and hemor rhage, particularly in the very small devices and in the very small EXCOR devices, so our overall outcomes are very similar. We do have some management differences, and that is going to be the focus of my question.

We rarely need to use BiVAD support. We have a very similar patient mix and primarily cardiomyopathies, and in 33 patients we have only needed to use BiVAD support twice. We place an LVAD in the operating room, attempt to wean from bypass, usually with inhaled nitric oxide, and if we do not require excessive inotro pic support, we have been very successful and continue with that.

What are your criteria for a BiVAD? How do you decide? Are you continuing to use a lot of BiVAD support?

Dr Chen. Thank you. Excellent question. Our management is very similar to yours insofar as we would always like to try to get away with one pump only, if only because more pumps just mean more potential for complications.

Earlier in our series we were seeing more children who were re ally in shock and going to the operating room while undergoing chest compressions. Those children are the least likely to come away with a single sided device only. As we have gotten more comfortable and more aggressive, the likelihood of catching pa tients earlier who will have reasonable right ventricular function is good.

I also think that in the later part of this experience we were using many fewer pulsatile, fill to empty left ventricular inflow devices. None of these, the centrifugal or axial flow devices, decompresses the ventricle quite as much and probably causes as much septal shift. So more recently I am hoping, as you point out, that we will be using fewer and fewer BiVADS and more and more LVADs as long we can effectively manage some right heart failure medically.

Dr Gaynor. You also mentioned that you switched to atrial can nulation. We have continued to use primarily apical cannulation except in patients with restricted physiology, and we believe that we get better decompression of the ventricle. We have not seen ma jor problems with the septal shifting. When would you use atrial cannulation now, if ever?

Dr Chen. That is a relatively new finding for us. It has been prompted a little bit by the hemorrhage/thrombosis component, which is that putting in the 2 atrial cannulas is very easy off pump. With this strategy, you can get away with half dose heparin and the bleeding is trivial in the operating room.

The disadvantage, of course, is that the decompression of the ventricle is less effective. Also, there is a possibility that if the aortic valve does not open, these children will have stasis in the left ven tricle and later clot if we do not start fairly aggressive anticoagula tion quickly enough. Some of the devices, of course, cannot be inserted atrially, but it is a much simpler approach if it is possible.
Dr Gaynor. I have 1 final question from the manuscript, which you did not address in the presentation, concerning the manage ment of patients who have intracardiac shunts. We always do a very careful search for intracardiac shunts, and we have enclosed them if we know we have them. We had 1 patient with an undiag nosed patent foramen ovale who was significantly desaturated when he came off LVAD support. You mentioned in the manuscript that with this off pump technique you will leave patients with intracardiac shunts and have not seen that problem. Could you expand on that?

Dr Chen. It was really more out of good luck, I think, than any thing. Regarding the intracardiac shunt component, a lot of these newer continuous flow devices do not decompress the ventricle enough that we get as much right to left shunting as we used to with their fill to empty predecessors. I was very concerned in $1 \mathrm{pa}$ tient, the smallest patient here, who had a BiVAD and multiple ventricular septal defects, that it was going to be hard to control the degree of shunting. However, it ended up not being so much of a problem. Atrial cannulation can be tricky with regard to atrial communications, though, and I suspect those probably still need to be closed.

Dr Gaynor. This answer brings up a further question. Most of our cases are bridged to transplant; we almost never bridge to re covery. We are always concerned that children frequently come in with high left atrial pressures and pulmonary edema, and we are concerned about dropping that left atrial pressure to improve the lungs. If you are using a device that does not decompress, will the atrial cannulation alone drop the left atrial pressure sufficiently to allow the lungs to recover?

Dr Chen. I do not know the answer to that. I would assume that the atrial cannulation probably will. I certainly do not think ECMO does though. It has definitely been our policy that if you are bridg ing to transplant with ECMO and you suspect high atrial pressures, you have to go to the catheterization laboratory for a septostomy at the time of instituting ECMO support, or very soon thereafter.

Dr Carl L. Backer (Chicago, Ill). For the patients whom you are supporting with ECMO, what is your decision tree for where to do the cannulation neck, femoral, or median sternotomy? We recently had an older child who came in with myocarditis. I put her on ECMO through the chest and she came off ECMO and went home after she was treated. The advantage of this in the older child is that there are no femoral or neck vessel compli cations. When these patients come in, it seems that there is always some discussion about where to do the ECMO cannulation. What is your strategy?

Dr Chen. For the smaller children and our cutoff ends up be ing about 2 years we go on through the neck. The hard group is that between about $5 \mathrm{~kg}$ and about $15 \mathrm{~kg}$, because we have had a fair number of groin complications with that size range, even with the T'd off sidearm that goes through the dorsalis pedis. When the patients get to be older and bigger, you can easily go with 2 venous cannulas, neck and groin, and femoral artery. It is just that middle group, I think, that is probably the right group to be having a sternotomy because it is much simpler and the vascular complications are substantial.

Dr Hajime Ichikawa (Osaka, Japan). I am really interested in your atrial cannula. We used to use an atrial cannula with Japanese Toyobo LVADs and we had a lot of problem with thrombosis. 
Do you change the anticoagulant regimen when using an atrial cannula?

Dr Chen. May I ask a question? Is the thrombosis in the pump or in the ventricle?

Dr Ichikawa. In the ventricle.

Dr Chen. We have worried a lot about thrombosis in the ventricle. We definitely run PTTs pretty high on all of these children; especially in the smaller children, we will run the PTTs well above 100. This is not necessarily based on the flow in the ventricle and so forth, but just as a general policy.

Dr Shunji Sano (Okayama, Japan). When I look at your table, the average duration is relatively short compared with the Toyobo device. The Toyobo device is completely different, almost 1 year. But most of these patients are relatively large children. So even with a Toyobo device, you use the adult size device. Why is the duration long and why was there only 1 episode of thromboembolism?

When I look at the small child, in this case comprising only 3 patients -1 with a Berlin Heart, 1 with the PediMag, and 1 with the Abiomed-they weighed less than $10 \mathrm{~kg}$. The longest duration was 17 days. How long do you think it is safe to wait for transplant with these 3 devices in very small babies weighing less than 10 $\mathrm{kg}-1$ month or 6 months or 1 year?

Dr Chen. The answer as to why that waiting time was so long for the Toyobo patients is that, as you know, children in Japan have to wait a fair amount of time for a potential donor. Each US program can only have $5 \%$ of their transplant volume as comprised of foreign national candidates, so sometimes these international children have to wait for the calendar year to turn over before we can accept them. There can be quite a bit of time for waiting and it takes time to raise money and so forth

Early in our experience we used the adult CentriMag circuit rather than the PediMag; that is a $3 / 8$-inch connection all the way around. It is actually a fairly large priming volume. A lot of children who were just over $10 \mathrm{~kg}$ in this group, about 11 to 13 $\mathrm{kg}$, would form a lot of early thrombus in the tubing, and so I started adapting to exchanging the tubing routinely at 2 weeks. We carried some of those patients up to about 6 weeks.

I do worry, though, when we put the smallest children on devices. We really try to get the transplant done as quickly as possible. The good news is that most of them can receive transplants across $\mathrm{ABO}$ blood groups because of their age. 